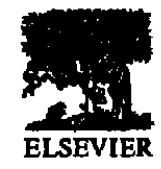

\title{
DELIPIDATING IN VITRO-PRODUCED BOVINE ZYGOTES: EFFECT ON FURTHER DEVELOPMENT AND CONSEQUENCES FOR FREEZABILITY
}

\author{
C. Diez, ${ }^{1 \mathrm{a}}$ Y. Heyman, ${ }^{1}$ D. Le Bourhis, ${ }^{2}$ C. Guyader-Joly, ${ }^{3}$ J. Degrouard, ${ }^{4}$ and J.P. Renard ${ }^{1}$ \\ 'INRA, Unité de Biologie du Développement, Jouy en Josas, France \\ ${ }^{2}$ UNCEIA, Maisons-Alfort, France \\ ${ }^{3}$ UNCEIA, Laboratoire FIV Chateauvillain, France \\ ${ }^{4}$ INRA, Biologie Cellulaire et Moléculaire Jouy en Josas, France
}

Received for publication: April 6, 2000

Accepted: September 29, 2000

\begin{abstract}
To study the effect of partial removal of intracytoplasmatic lipids from bovine zygotes on their in vitro and in vivo survival, presumptive zygotes were delipidated by micromanipulation and cocultured with Vero cells in $\mathrm{B}_{2}+10 \%$ FCS. Blastocyst rates of delipidated $(n=960)$, sham (centrifuged but not delipidated, $n=830$ ) and control embryos $(n=950)$ were $42.1,42.3$ and $39.9 \%$ respectively $(\mathrm{P}>0.05)$. Day 7 blastocysts derived from delipidated zygotes had a mean of 123.9 \pm 45.6 nuclei compared to $137.5 \pm 32.9$ for control blastocysts $(P>0.05)$. The full-term development of delipidated blastocysts after single transfer to recipients was similar to that of control IVF blastocysts ( $41.2 \%$ vs $45.4 \%$ respectively). To assess the effect of delipidation on the embryo tolerance to freezing/thawing, delipidated $(n=73)$, control $(n=67)$ and sham $(n=50)$ Day 7 blastocysts were frozen in $1.36 \mathrm{M}$ glycerol $+0.25 \mathrm{M}$ sucrose in PBS. After thawing, embryos were cocultured for $72 \mathrm{~h}$ with Vero cells in $\mathrm{B}_{2}+10 \%$ FCS. Survival rates at $24 \mathrm{~h}$ were not significantly different between groups. However, in the delipidated group, the survival rate after $48 \mathrm{~h}$ in culture was significantly higher than in the control group (56.2 vs $39.8, \mathrm{P}<0.02$ ), resulting in a higher hatching rate after 3 days in culture (45.2 vs $22.4, \mathrm{P}<0.02)$. Pregnancy rates for delipidated and control frozen/thawed embryos were respectively 10.5 and $22.2 \%(\mathrm{P}>0.05)$. Electron microscopic observations showed much fewer lipid droplets (and smaller) in delipated blastocysts than in controls. Taken together, our data show that delipidation of one cell stage bovine embryos is compatible with their normal development to term and has a beneficial effect on their tolerance to freezing and thawing at the blastocyst stage. This procedure, however, alters the developmental potential of such blastocysts, suggesting that maternally inherited lipid stores interfere with metabolic recovery after thawing.

- 2001 by Elsevier Science Inc.
\end{abstract}

Key words: IVP bovine embryos, bovine, freezing, lipids

Acknowledgments

The authors thank Mrs. C. Menck and Mrs. N. Peynot for help in the in vitro laboratory, Mr. J. Marchal and the staff of the experimental farm INRA-Bressonvillers and Dr. P. Adenot for his help in the use of confocal microscopy.

${ }^{2}$ Correspondence address: C. Díez. SERIDA-CENSYRA. Camino de los Claveles s/n 33203 Somio, Gijón (Asturias). Spain. Phone: 34 985195300. Fax: 34985195310 e-mail: carmendm@princast.es 


\section{INTRODUCTION}

In the cattle embryo transfer industry, embryos increasingly are produced totally in vitro from immature oocytes that are collected by repeated ovum pick-up or from slaughterhouse ovaries; then matured, fertilized and cultured in vitro up to the blastocyst stage (for review see: $14,18)$. Efficient utilization of in vitro-produced embryos requires that they are able to be frozen before their transfer later in different locations. This is the case for in vivo produced bovine blastocysts, which are efficiently stored in liquid nitrogen as currently about $51 \%$ of the embryo transfers in cattle are performed using frozen-thawed blastocysts (1).

However, in vitro produced (IVP) embryos are more sensitive to chilling (31) and freezing than their in vivo counterparts $(16,20)$. The differences between in vitro and in vivo produced embryos have been investigated by several authors $(13,36)$. The composition of culture medium and particularly the presence of serum, has a marked influence on morphology and cytoplasmic appearance of in virro-produced embryos that look dark. Under close examination, these blastocysts have numerous "granules" in the trophectoderm cells (12,37). Pollard and Leibo (31) noticed that a difference in the zona pellucida to enzymatic digestion between in vivo and in vitro produced embryos could modify their permeability to water and cryoprotective agents. Furthermore, Leibo and Loskutoff (16) using sucrose solutions of different gravity, demonstrated that the buoyant density of IVP bovine embryos is lower than that of the in vivo ones. According to these authors, the possible explanation for this difference in buoyant densities is that the ratio of lipids/protein in the cytoplasm of IVP embryos is greater than that of in vivo embryos. The exact role those lipids can play with respect to the cryo-sensitivity is not known. In the pig, it was clearly demonstrated that removal of cytoplasmic lipids by micromanipulation at the 1-cell stage after centrifugation, enhanced the tolerance of embryos to chilling and freezing $(25,27)$ or vitrification (28). Our preliminary experiments (5), together with the results of Ushijina et al. (38) showed that lipid removal from in vitro produced one-cell stage zygotes was compatible with their further in vitro development. However, it is important to investigate the development potential of partially delipated embryos and their sensitivity to cryopreservation.

In the present experiment, we studied the effect of the removal of intracytoplasnic lipids from bovine zygotes on their in vitro and in vivo survival, and we assessed the effect of delipidation on their freezing tolerance at the blastocyst stage.

\section{MATERIALS AND METHODS}

\section{Preparation of IVM//VF Embryos}

Cow ovaries were collected at a local slaughterhouse and transported to the laboratory in saline at $30^{\circ} \mathrm{C}$ within $3 \mathrm{~h}$. Immature oocytes were aspirated from antral follicles $(2 \mathrm{to} 7 \mathrm{~mm}$ in diameter) with a $191 / 2 \mathrm{~g}$ needle. Cumulus oocyte complexes (COCs) with more than three compact layers of cumulus cells were selected, pooled and rinsed three times in fresh medium before maturation. Maturation medium consisted of $25 \mathrm{mM}$ bicarbonate-buffered TCM199 (Gibco, Grand Island, New York, USA) supplemented with $10 \%(\mathrm{v} / \mathrm{v})$ heat inactivated fetal calf serum (FCS) (Dutscher, Brumath, Germany), $10 \mu \mathrm{g} \mathrm{FSH} / \mathrm{mL}, 10 \mu \mathrm{g} \mathrm{LH} / \mathrm{mL}$ (Merial, Lyon, France) and Estradiol $17 \beta 1 \mu \mathrm{g} / \mathrm{mL}$ (Sigma, St. Louis, MO, USA). Then, the oocyles were cultured in maturation medium for $24 \mathrm{~h}$ at $39^{\circ} \mathrm{C}$ under a humidified atmosphere of $5 \% \mathrm{CO}_{2}$ in air. 
At the end of the maturation period, the oocytes were fertilized in vitro in TALP (Tyrode modified) medium supplemented with $1 \mu \mathrm{g}$ heparin $/ \mathrm{mL}$ (Sigma), and PHE (penicillamine, hipotaurine, epinephrine). Spermatozoa separated by the swim-up method were added at a concentration of $1 \times 10^{6}$ spermatozoa/mL. The day of insemination was referred to as Day 0 , and the same batch of frozen spermatozoa (frozen straws from the same bull and ejaculate) was used throughout the experiment. Eighteen hours after insemination, COCs were removed from the fertilization medium and presumptive zygotes were denuded from cumulus cells by vortexing in M199 $26 \mathrm{mM}$ Hepes buffered+10\% FCS for $3 \mathrm{~min}$. After washing in culture medium $\left(\mathrm{B}_{2}+10 \%\right.$ FCS), for each replicate experiment, presumptive zygotes were assigned randomly to three groups: delipidated zygotes, sham manipulated or intact controls.

\section{Lipid Removal}

The micromanipulation method used in this work was originally published by Nagashima et al. (25). To polarize cytoplasmic lipid granules within the cell, presumptive zygotes (19 h post insemination) were centrifuged at room temperature for $7 \mathrm{~min}$ at $15800 \mathrm{~g}$, in $\mathrm{M} 199$ Hepes containing $10 \% \mathrm{FCS}$ and a cytoskeletal inhibitor ( $5 \mu \mathrm{g}$ Cytochalasin B/mL, Sigma) in $1.5 \mathrm{~mL}$ microcentrifuge tubes. Centrifugated zygotes were allowed to stay for 30 to $45 \mathrm{~min}$ in culture medium; then, the lipid plug was removed by micromanipulation. A holding pipette maintained the zygote in position and a beveled suction pipette ( 30 to $40 \mu \mathrm{m}$ in diameter) connected to a micromanipulator was introduced through the zona to aspirate the lipid plug (Figure la). Manipulations were performed under an inverted microscope (Olympus IMT2) at X 200 magnification. After lipid removal, the delipidated embryos were washed three times in M199 Hepes $+10 \%$ FCS and once in $\mathrm{B}_{2}+10 \%$ FCS and were placed in coculture.

\section{In Vitro Culture}

In vitro development of the different groups of zygotes (delipidated, sham or controls) up to the blastocyst stage was achieved using the coculture technique on Vero cells currently used in our laboratory for IVF embryos (23). Briefly, groups of 20 to 25 zygotes were placed in drops of $50 \mu \mathrm{L} \mathrm{B} \mathrm{B}_{2}$ medium supplemented with $10 \% \mathrm{FCS}$, seeded with Vero cells and overlayed with mineral oil. Cocultures were performed at $39^{\circ} \mathrm{C}$ under $5 \% \mathrm{CO}_{2}$ in air for seven days. Eggs were observed daily up to Day 8 and were evaluated for cleavage and blastocyst formation. To evaluate fertilization rate, zygotes that were not cleaved at $24 \mathrm{~h}$ of coculture were removed and stained with Hoechst dye 33342 (Sigma) and observed under an inverted microscope equipped with epifluorescence for the presence of pronuclei. The fertilization rate was calculated from the number of cleaved eggs plus the number of non cleaved eggs that contained 2 pronuclei, related to the number of inseminated oocytes.

\section{Embryo Transfer}

To assess further in vivo viability of delipidated embryos, blastocysts were transferred to the uterus of synchronous recipient heifers (one blastocyst per recipient, nonsurgically transferred to the uterine horn ipsilateral to the corpus luteum). Pregnancy diagnosis was first assessed by a plasma progesterone test on Day 21 and then confirmed by ultrasonography on Days 35, 50 and 90. Pregnant recipients were kept until calving and the number of offspring was recorded 


\section{Freezing Procedure}

In vitro-produced Day 7 blastocysts were frozen according to the protocol of Massip et al. (1993), used for in vivo-produced embryos. Our purpose was to submit the different groups of IVP embryos to the same extreme conditions during the freezing process, and to make the differences among the groups more evident. Embryos were equilibrated in $1.36 \mathrm{M}$ glycerol + $0.25 \mathrm{M}$ sucrose in PBS at room temperature for 15 to $20 \mathrm{~min}$ and loaded into $0.25 \mathrm{~mL}$ straws (IMV L'Aigle, France) placed into a programmable freezing machine and cooled from 20 to $-7^{\circ} \mathrm{C}$ at a rate of $5^{\circ} \mathrm{C} / \mathrm{min}$. The straws were seeded at $-7^{\circ} \mathrm{C}$, and then cooled at a rate of $0.3^{\circ} \mathrm{C} / \mathrm{min}$ to $-30^{\circ} \mathrm{C}$. Finally, they were plunged into and stored in liquid nitrogen. For thawing, the straws were plunged into a $20^{\circ} \mathrm{C}$ water bath for $30 \mathrm{sec}$. The embryos were expelled in a solution of 0.25 M sucrose in PBS with 10\% FCS for 5 min and then washed in PBS $+20 \%$ FCS for another 5 min before coculture for 3 days to assess further in vitro development and hatching. Embryos were evaluated microscopically at $24 \mathrm{~h}$ intervals for $72 \mathrm{~h}$. Survival rates were assessed by (a) reappearance of the blastocoele cavity, (b) attainment of a fully expanded stage and (c) ability to hatch in vitro.

\section{Experimental Design}

Experiment 1: Effect of lipid removal on in vitro and in vivo survival. For this experiment presumptive zygotes from the same batch of IVF were assigned randomly to 3 groups: 1) Delipidated group: cytoplasmic lipids were removed by micromanipulation as described above. 2) Sham group: zygotes were treated with Cytochalasin B and centrifuged but were not micromanipulated, to assess the effect of this treatment: after centrifugation, they were rinsed and cocultured as delipated embryos. 3) Control group: intact presumptive zygotes were directly cocultured in vitro without any other manipulation.

To assess in vitro development for the 3 groups, the cleavage rate was recorded on Day 2 of culture ( $42 \mathrm{~h}$ post insemination). The number of embryos developing to the blastocyst stage was assessed on Days 6 to 8 of culture. Representative samples of delipidated and control embryos were fixed at the end of Day 2 (four-cell stage) and by Day 7 (blastocysts) for ultrastructure assessment by electron microscopy. Quality of Day 7 blastocysts derived from delipidated or control zygotes was assessed also by counting nuclei, by using propidium iodide staining and by confocal microscopy (15). To confirm the in vivo developmental competence of "lipid free" blastocysts, embryo transfers were made to synchronous recipient heifers.

Experiment 2: Effect of lipid removal on freezing tolerance. Day 7 blastocysts developed after lipid removal at the zygote stage were frozen-thawed and their survival was assessed in vitro and compared to that of sham or control embryos frozen in the same conditions. To evaluate the full-term developmental ability of "lipid free" frozen/thawed blastocysts, embryo transfers were made (within one hour after thawing) to synchronous recipient heifers which were kept until calving. 


\section{Statistical Analysis}

The effect of the treatment on in vitro development of embryos (cleavage and blastocyst rates) was analyzed by a chi-square test $(\chi 2)$ for $2 \times 3$ contingency tables. A significant overall chi-square test for a $2 \times 3$ contingency table indicates the differences among the three groups (control, delipidated and sham). To provide information for the significant groups we subdivided the overall chi-square value into two components parts, obtained by partitioning the tables into nonindependent $2 \times 2$ tables (control vs delipidated, control vs sham and delipidated vs sham). For each table we compared the chi-square value against the one degree of freedom value from the tables. The level of significance was reduced from 0.05 to 0.02 after the application of Buonferroni correction for multiple comparisons ( 3 comparisons).

\section{RESULTS}

\section{Experiment 1}

A total of 960 presumptive zygotes were delipidated by micromanipulation and then cultured in vitro. Results of cleavage and blastocyst formation are presented in Table 1 and compared to results of $\mathbf{8 3 0}$ sham embryos and 950 controls. No significant difference was observed between groups either for cleavage or blastocyst rate indicating that the removal of most of the cytoplasmic lipid content after centrifugation in bovine zygotes did not impair their in vitro developmental potential up to the blastocyst stage. The blastocyst rates were respectively 42.1 , 39.9 and $42.3 \%$ for delipidated, sham and control embryos. By Day 7, blastocysts derived from delipidated zygotes had a mean number of $123.9 \pm 45.6$ nuclei compared to $137.5 \pm 32.9$ for blastocysts in the control group, the difference being not significant. Microscopic pictures clearly showed the difference in cytoplasmic darkness at 2 or 8 cell stages between delipidated and control embryos (Figures 1b, 1c). Ultrastructural observations on delipidated embryos (Figures $2 \mathrm{a}, 2 \mathrm{~b}$ ) clearly indicate that very few lipid droplets were present in the cytoplasm of 4 cell stage and blastocyst stage compared to control nondelipidated embryos developed in vitro under the same conditions.

Table 1. In vitro development of delipated, sham and control embryos.

\begin{tabular}{lcccc}
\hline & Replicates & $\begin{array}{c}\text { Presumptive } \\
\text { zygotes }\end{array}$ & $\begin{array}{c}\text { Number cleaved } \\
(\%) \mathrm{a}\end{array}$ & $\begin{array}{c}\text { Number of } \\
\text { blastocysts (\%)a }\end{array}$ \\
\hline Delipidated & 20 & 960 & $892(95.8)^{\mathrm{b}}$ & $392(42.1)^{\mathrm{c}}$ \\
Sham & 20 & 830 & $759(94.3)^{\mathrm{b}}$ & $321(39.9)^{\mathrm{c}}$ \\
Control & 21 & 950 & $868(94.3)^{\mathrm{b}}$ & $390(42.3)^{\mathrm{c}}$
\end{tabular}

\footnotetext{
Cleavage and blastocyst rates were calculated related to the number of oocytes fertilized. Average rate of fertilization was $97 \%$.

bc Columns with different superscripts differ significantly,
} 
a

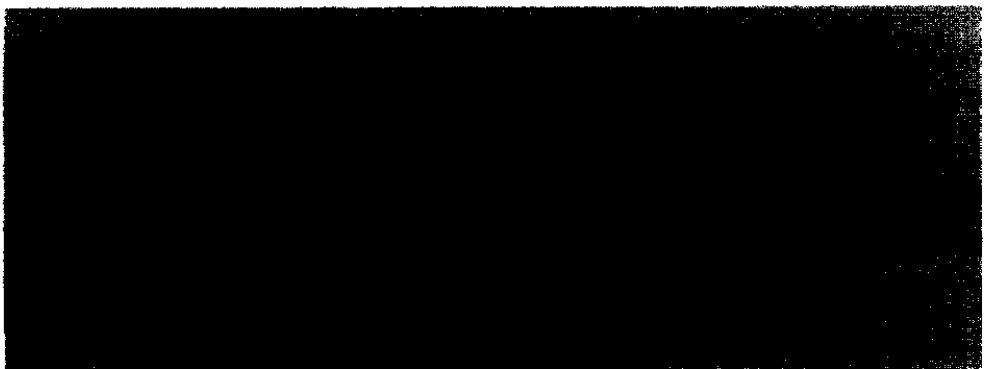

b

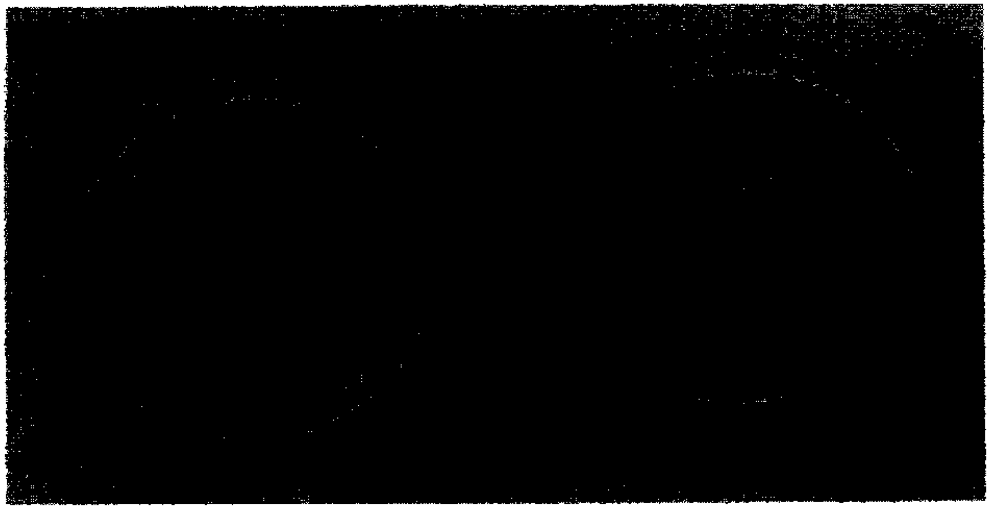

C

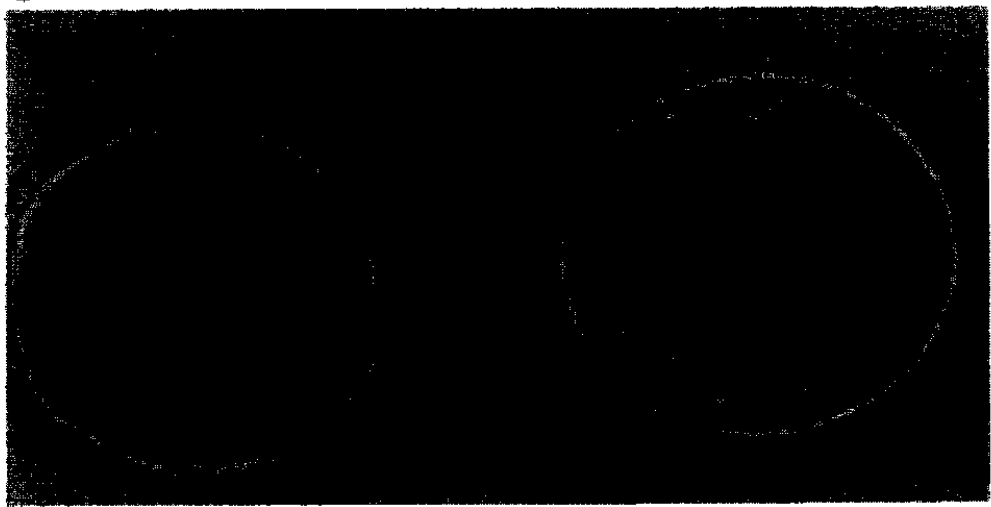

Figure 1. Delipidating bovine embryos. a) Presumptive zygote after centrifugation and before removal of the lipid plug. b) Control (left) and delipidated (right) two cell-stage embryo. c) Control (left) and delipidated (right) eight cell-stage embryo. 
a

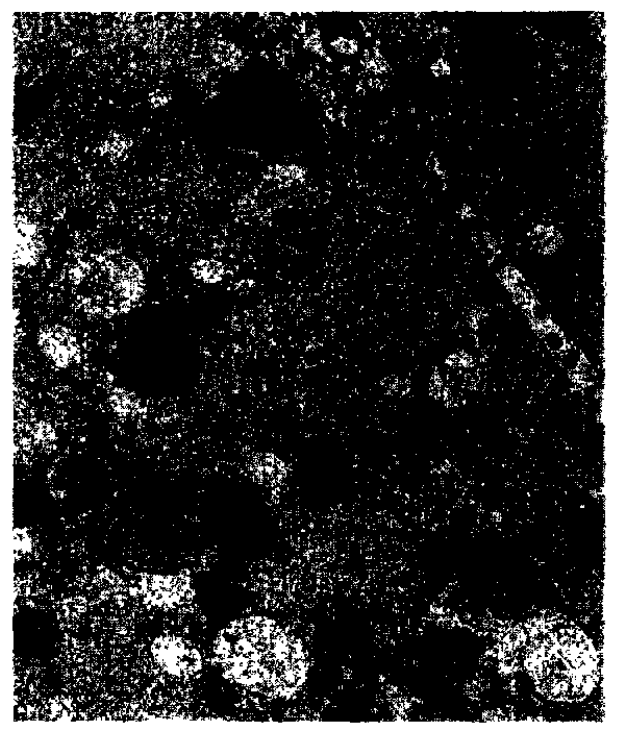

C

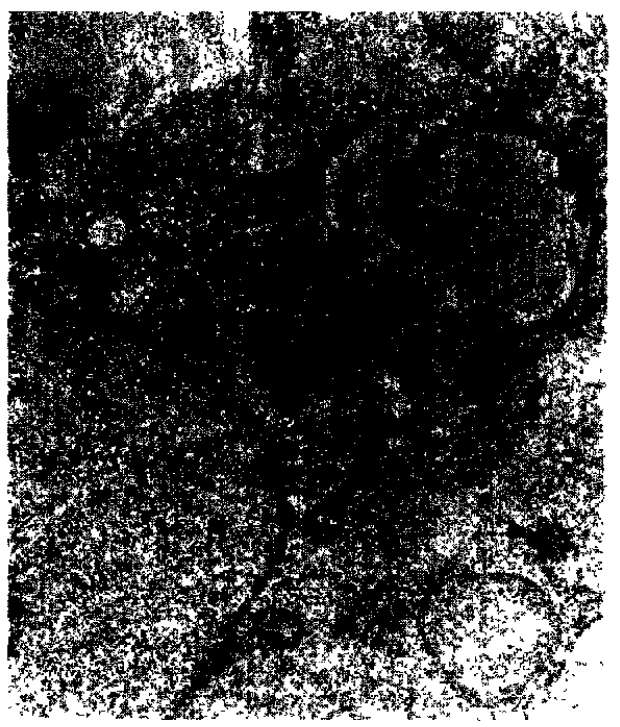

b

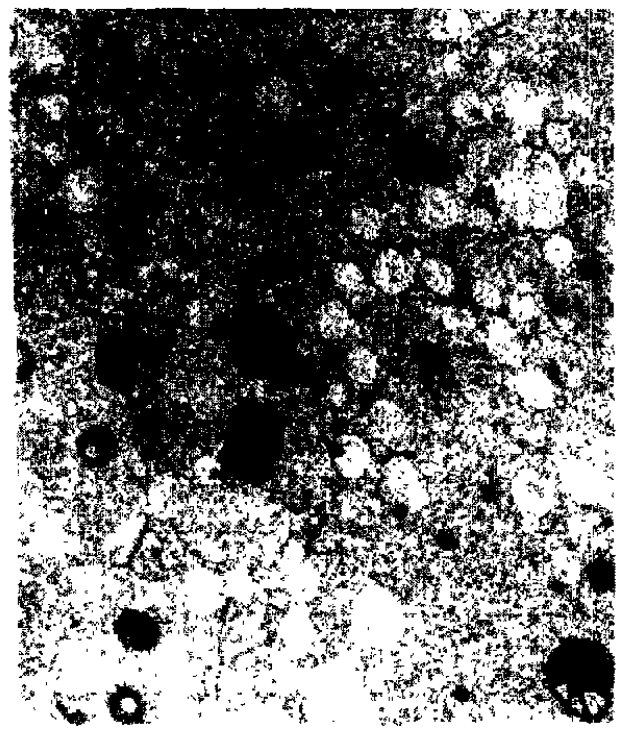

d

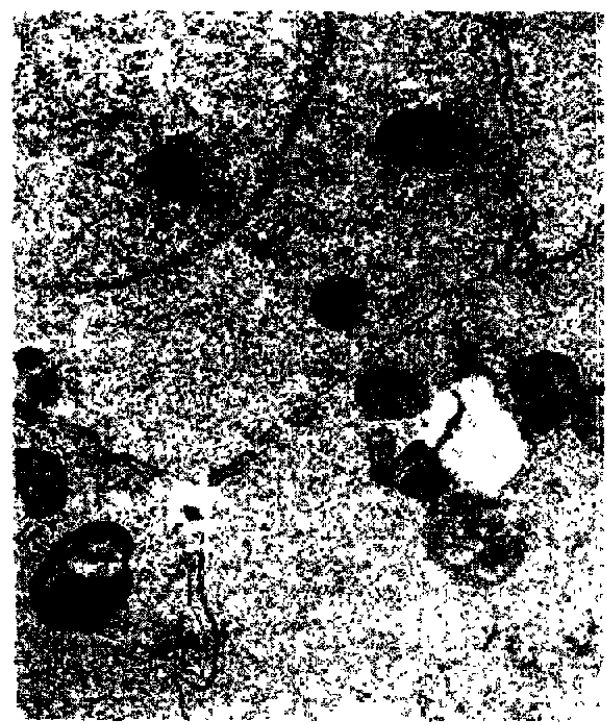

Figure 2. TEM micrographs ( $\mathrm{X}=11500)$. a) Control in vivo bovine embryo at the 4-cell stage. Note important lipid inclusions (arrows) in the cytoplasm of the blastomere. b) Section of 4-cell stage embryo after delipidation. No apparent lipid inclusions are visible in the cytoplasm. c) ICM of control blastocyst. Arrows indicate large inclusions of lipids droplets. d) ICM of blastocyst after lipid removal. Note the limited presence of small lipid droplets in the cytoplasm (arrows). 
The developmental competence of delipidated blastocysts was assessed by transferring 17 embryos to synchronous recipient heifers (1 embryo/recipient). Eleven recipients (64.7\%) established pregnancy as confirmed by positive plasma progesterone test on Day 21 (Table 2), and $7(41.2 \%)$ were confirmed pregnant on Day 90 by ultrasonography. These recipients gave birth to eight healthy calves (one recipient delivered twin calves). The full-term development of delipidated blastocysts was similar to that of control IVF blastocysts transferred to the control group of recipient (10 calves born from 22 embryos, $45.4 \%$ ).

Table 2. In vivo survival of blastocysts developed from delipidated zygotes

Number of pregnancies (\%)

\begin{tabular}{lccccc}
\hline & & P4 test & \multicolumn{3}{c}{ Ultrasonic echography } \\
\hline Group & Recipients & D 21 & D 35 & D90 & Calves born \\
\hline $\begin{array}{l}\text { Delipidated } \\
\text { blastocysts }\end{array}$ & 17 & $11(64.7)$ & $9(52.9)$ & $7(41.2)$ & $8(47.0)^{\mathrm{n}}$ \\
\hline $\begin{array}{l}\text { Control IVF } \\
\text { blastocysts }\end{array}$ & 22 & $13(59.0)$ & $11(50.0)$ & $10(45.4)$ & $10(45.4)$ \\
\hline
\end{tabular}

'One recipient transferred with a single blastocyst gave birth to a pair of monozygotic twins. Chi-square test: no significant difference was found.

\section{Experiment 2}

A total of 73 blastocysts derived from delipidated zygotes were frozen-thawed and their survival was assessed in vitro and compared to that of blastocysts developed from sham or control zygotes. Results are presented in Table 3 . Survival rates at $24 \mathrm{~h}$ were not significantly different between groups. However in the delipidated group, survival rate after $48 \mathrm{~h}$ in culture was significantly higher than in the control group ( 56.2 vs $39.8, \mathrm{P}<0.02)$, resulting in a higher hatching rate after three days in culture $(45.2$ vs $22.4, \mathrm{P}<0.02)$. Interestingly, frozen blastocysts in the sham group, in which lipids were concentrated by centrifugation at the zygotic stage but not removed, showed intermediate survival between delipidated and control embryos.

Table 3. In vitro survival of delipidated, sham and control frozen/thawed blastocysts

\begin{tabular}{lcccc}
\hline & \multicolumn{3}{c}{ Number developing in vitro (\%) } & Hatching \\
\cline { 2 - 5 } & $\mathrm{n}$ & $24 \mathrm{~h}$ & $48 \mathrm{~h}$ & $72 \mathrm{~h}$ \\
\hline Delipated & 73 & $37(50.7)^{\mathrm{a}}$ & $41(56.2)^{\mathrm{a}}$ & $33(45.2)^{\mathrm{a}}$ \\
\hline Sham & 50 & $16(32.0)^{\mathrm{a}}$ & $20(40.0)^{\mathrm{a}, 0}$ & $15(30.0)^{\mathrm{a}}, \mathrm{s}$ \\
\hline Control & 67 & $21(31.3)^{\mathrm{a}}$ & $22(39.8)^{\mathrm{a}}$ & $15(22.4)^{\mathrm{\sigma}}$ \\
\hline
\end{tabular}

${ }^{\mathrm{abc}}$ Columns with different superscripts differ significantly $(\mathrm{P}<0.02)$. 
The developmental competence of delipidated frozen/thawed blastocysts was assessed by transferring 19 embryos to synchronous recipient heifers ( 1 embryo/recipient) and compared to that of control IVF blastocysts frozen under the same conditions. Results are given in Table 4 . From the delipidated group, six recipients (31.5\%) initiated pregnancy as confirmed by positive plasma progesterone test on Day 21 , but only two (10.5\%) were confirmed pregnant on Day 90. These recipients gave birth to two healthy calves.

Table 4. In vivo survival of delipated blastocysts after freezing/thawing

\begin{tabular}{lccccc}
\hline & & \multicolumn{4}{c}{ Number of pregnancies (\%) } \\
\cline { 3 - 6 } & & P4 test & \multicolumn{3}{c}{ Ultrasonic echography } \\
\hline Group & Recipients & D 21 & D 35 & D90 & Calves born \\
\hline $\begin{array}{l}\text { Delipidated } \\
\text { trozen }\end{array}$ & 19 & $6(31.5)$ & $3(15.8)$ & $2(10.5)$ & $2(10.5)$ \\
\hline $\begin{array}{l}\text { Control } \\
\text { trozen }\end{array}$ & 18 & $7(38.9)$ & $6(33.3)$ & $4(22.2)$ & $4(22.2)$ \\
\hline
\end{tabular}

Chi-square test: no significant difference was found.

\section{DISCUSSION}

According to the present experiments, centrifugation and removal of most of the cytoplasmic lipid plug at the zygote stage did not affect the in vitro developmental competence of the manipulated bovine embryos. Sham and delipidated embryos cleaved and developed in a similar way to the control group, with a blastocyst rate that varied around $40 \%$. This indicates that the method used for micromanipulation was not detrimental for subsequent in vitro development. In our protocol, we estimate that approximately $90 \%$ of the dense lipid fraction after centrifugation was removed from the zygote, which is very similar to the observations on porcine embryos by Nagashima et al. $(25,26,27)$ in which fully delipidated one cell eggs had the same developmental potential than intact controls. So, delipidated zygotes are able to develop in the absence of lipids normally found in one cell stage.

Centrifugation itself in the presence of cytochalasin (sham embryos) had no negative effect on in vitro survival of embryos, provided that the centrifuged zygotes were not manipulated immediately after centrifugation but only after a minimum of 30 to 45 min period in culture medium. Indeed in preliminary studies, we observed that micromanipulation immediately after centrifugation was associated with increased lysis. Displacement of cytoplasmic structures by centrifugation probably needs a short period of recovery for stabilization of the membranes. This could explain our higher blastocyst rate (42\%) compared to that (12\%) of Pangestu et al. (30) who concluded that removal of the intracytoplasmic lipids perturbed the in vitro development to the blastocyst stage. Cytochalasin B renders the plasma membrane less rigid and more elastic (22). This cytoskeletal stabilizer can be used reversibly to depolymerise actin microfilaments in bovine (6) and porcine (7) embryos before vitrification and then to improve their in vitro 
survival rate after thawing. Depolymerization of microfilaments can enhance their ability to repolymerize after thawing during dilution and rehydration periods.

At early stages, stereomicroscope pictures clearly showed the difference in cytoplasmic darkness at the 2-or 8-cell stage between delipidated and control embryos (Figures 1b,1c). This was confirmed by electron microscopic observations (Figures $2 \mathrm{a}, \mathrm{b}, \mathrm{c}$ and d). At these stages and at the blastocyst stage, electron micrographs showed far fewer lipid droplets in delipidated blastocysts than in controls; furthermore, these droplets were of small size. These observations suggest that during in vitro culture there is a lipid accumulation in cytoplasm. The content and function of cytoplasmic lipid droplets is not well known, but Flynn and Hillman $(10,11)$ showed that preimplantation mouse embryos are able to synthesize lipids de novo from exogenous glucose or fatty acids present in the microenvironment. Neutral lipids such as triacylglycerol are the major lipid species synthesized and stored. A major biological function of triacylglycerols is to serve as a storage form of metabolic energy in preparation for specific developmental events such as the hatching of the blastocyst (10). In embryos of different species, mitochondria have been colocalized with lipid droplets but the functional significance of these associations is unclear $(8,29,39)$. They are thought to provide a reserve of lipid for oxidation by mitochondrial oxidases. Dorland et al. (8) observed, in ovine embryos cultured in SOF medium supplemented with serum, a high degree of mitochondrial degeneration compared to those cultured in medium supplemented with amino acids. The number and size of lipid droplets was also larger in embryos developed in serum-supplemented medium. They concluded that under culture conditions that caused degeneration of mitochondria in sheep embryos, lipid droplets increased in size, implying impaired lipid metabolism when mitochondria are damaged. Maybe a "defined system" for producing embryos without using substrates obtained from FCS or BSA to produce intracytoplasmic lipids would allow us to understand more aspects of this problem.

Both the in vitro developmental ability of delipidated zygotes and the morphologic normality of resulting the blastocysts in terms of number of cells and ultrastructure could explain the high in vivo survival into full-term calves ( 8 of $17 ; 47 \%$ ), similar to that obtained after transfer of control IVF blastocysts (45.4\%). In this work, the birth of a set of twins after the transfer of a single delipidated blastocyst is due to natural splitting during hatching, probably facilitated by fragilization of the zona during previous micromanipulation. Such cases of twinning after the transfer of a single embryo was previously reported by Massip et al. (19).

In the second experiment, delipidated, sham and control Day-7 blastocysts were frozen using a conventional slow freezing procedure (19). This protocol is used for in vivo-produced embryos and is not optimal for in vitro ones, but the purpose of this freezing thawing process was to submit the different groups of IVP embryos to the same extreme conditions during the freezing process, and to make the differences between the groups more evident. After freezing and thawing, delipidated embryos survived significantly better in vitro than control embryos and hatched at a higher rate. Our results on bovine embryos coincide with the ones reported in the pig by Nagashima et al. (27), who found significantly improved in vitro survival rates after freezing delipidated embryos. The same group reported successful vitrification of pig embryos after delipidation $(24,28)$. With bovine IVP embryos, Pangestu et al. (30) did not obtain survival of delipidated blastocysts after slow freezing. Using another approach, Leibo et al. (17) displaced 
cytoplasmic lipids by centrifugation in bovine zygotes, and reaggregated chimeric embryos made of "lipid poor" or "lipid rich" blastomeres. They achieved $40 \%$ survival after chilling and $33 \%$ after freezing "lipid poor embryos" compared to $0 \%$ after chilling or freezing "lipid rich embryos."

Frozen/thawed sham embryos had intermediate survival rates between delipidated and control embryos, without significant differences. Similarly, Nagashima et al. $(26,27)$ demonstrated that 1- to 4-cell stage centrifuged embryos were not as tolerant as delipidated embryos, but they had significantly higher survival rates than control embryos. In this work, only a part of the lipid droplets polarized by centrifugation could redistribute during in vitro development, producing blastocysts darker than delipidated embryos but clearer than the control ones, and retaining the rest of the droplets outside the cytoplasm. This different lipid content in the blastomeres could give to the embryos an intermediate sensitivity against the freezing process.

In the present experiment, the beneficial effect of lipid removal on the freezing tolerance that was observed in vitro was not confirmed after the transfer of the embryos to recipient heifers. This was because the full-term development into live calves of delipidated and frozen-thawed embryos was only $10.5 \%$ compared to $47.0 \%$ for nonfrozen ones. This could be partly due to the freezing procedure itself in which no serum was added to the freezing medium. It was observed that the presence of serum together with glycerol during freezing improves in vitro survival (Diez, C., unpublished data). Despite the limited numbers of frozen-thawed embryos transferred, it seems that there is no direct relationship between in vitro and in vivo survival of delipidated frozen-thawed embryos. From these results, we can conclude that in our in vitro culture conditions, developed embryos accumulate intracellular lipid droplets. A similar situation has been described in tissue culture cells which tend to form lipid inclusion bodies, containing triacylglycerols, when they are growing in lipid rich culture media containing either free fatty acid or plasma protein $(35,34)$. Moreover, many types of cells modify the composition of their membrane phospholipid fatty acids during short-term culture if the type of lipid in the culture medium is restricted or enriched with a particular fatty acid $(3,33)$. The presence or reducing the number of these droplets doesn't affect subsequent in vivo viability of fresh embryos after transfer. We can hypothesize that when fresh embryos are replaced under physiological conditions after transfer, they may regulate rapidly their lipid metabolism to restore fundamental cell functions. Intracellular lipid droplets may affect physical properties of cellular membranes when submitted to the freezing/thawing process as attested by the poor in vitro and in vivo survival rates of nondelipidated frozen blastocysts. Sensitivity to chilling may be due to irreversible changes in the membrane structure that occur when embryos are cooled below a critical temperature $(32,41)$. These changes have been attributed to lateral phase separations of the plasma and/or internal membrane lipids (32). In porcine embryos maintained at $15^{\circ} \mathrm{C}$, lipid droplets tend to coalesce to form larger droplets (9). By changing the lipid content of in vitrocultured bovine embryos, we obtained a higher in vitro survival rate after freezing. But the fact that in vivo survival rate was not increased leads to several unanswered questions. Are these lipids, present as droplets within the cytoplasm, essential for further cellular events implying blastocyst elongation or implantation process? Does the freezing/thawing procedure alter or reduce the potential of embryos to restore fundamental cell functions? The effects of lipid modification on cellular physiology and metabolic regulation are very complex. Further research 
is needed to determine the functional significance and composition of the cytoplasmic lipid granules.

\section{REFERENCES}

1. AETE Statistic Data of the bovine embryo transfer activity in Europe for 1998. In: Proceedings of the 15th AETE Scientific Meeting, Lyon 1999 pp 27-69

2. Aoyagi $\mathrm{Y}$, Konishi M, Takedomi T, Ikatura H, Itoh T, Yazawa S. Effect of lipid rich bovine serum albumin on direct transfer of frozen-thawed bovine embryos. Theriogenology 1996;45: 165 abstr.

3. Bailey JM, Dunbar LM Essential fatty acid requirements of cells in tissue culture : a review. Exp Mol Pathol 1973;18: 142-161.

4. Diez C, Guyader-Joly C, Ponchon S, Heyman Y, Renard JP. Freezing bovine IVP embryos: presence of serum and two step dilution improves in vitro. In: Proceedings of the 12th AETE Scientific Meeting, Lyon, 1996: pp. 28.

5. Diez C, Le Bourhis D, Heyman Y, Renard JP. Effect of partial lipid removal from bovine zygotes on further survival and freezing tolerance of in vitro-produced blastocysts. Theriogenology 1995; 45(1): 166 abstr.

6. Dobrinsky JR. Cellular approach to cryopreservation of embryos. Theriogenology $1996 ; 45(1): 17-26$.

7. Dobrinsky JR, Pursel VG, Long CR, Johnson LA. Birth of normal piglets after cytoskeletal stabilization of embryos and cryopreservation by vitrification. Theriogenology 1997; 49 (1): 166 abstr.

8. Dorland $M$, Gardner DK, Trounson AO. Serum in synthetic oviduct fluid causes mitochondrial degeneration in ovine embryos. J Reprod Fertil 1995; Abstr. Ser. 13: 70.

9. Edidin M, Petit VA. The effect of temperature on the lateral diffusion of plasma membrane protein. In: Elliott K. Whelan (eds.), The freezing of Mammalian Einbryos. Amsterdam: Elsevier, Excepta Medica 1977; 155-174.

10. Flynn T, Hillman N. Lipid synthesis from (U-14C) glucose in preimplantation mouse embryos in culture. Biol Reprod 1978; 19 : 922-926.

11. Flynn T, Hillman $\mathrm{N}$. The metabolism of exogenous fatty acids by preimplantation mouse embryos developing in vitro. J Embryol Exp Morph 1980; 56: 157-168.

12. Gardner DK, Lane M, Spitzer R, Batt P. Enhanced rate of cleavage and development for sheep zygotes cultured to the blastocyst stage in vitro in the absence of serum and somatic cells: amino acids, vitamins and culturing embryos in groups stimulate development. Biol Reprod 1994; 50: 390-400.

13. Greve T, Avery B, Callesen $\mathrm{H}$. Viability of in vivo and in vitro-produced bovine embryos. Reprod Domest Anim 1993; 28 (4): 164.

14. Hasler JF, Henderson WB, Hurtgen PJ, Jin ZQ, Mc Cauley AD, Mower SA, Neely B, Shuey LS, Stokes JA, Trimmer SA. Production, freezing and transfer of bovine IVF embryos and subsequent calving results. Theriogenology 1995; 43: 141-152.

15. Heyman Y, Degrouard J, Adenot P, Chesne P, Flechon B, Flechon JE. Cellular evaluation of bovine nuclear transfer embryos developed in vitro. Reprod Nutr Dev 1995; 35: 713 723.

16. Leibo SP, Loskutoff NM. Cryobiology of in vitro-derived bovine embryos. Theriogenology, 1993; 40: 81-94. 
17. Leibo SP, Pollard JW, Martino A. Chilling and freezing sensitivity of "reassembled" in vitro-derived bovine embryos. Theriogenology 1995; 43: 265 abstr.

18. Looney CR, Lindsay BR, Gonseth CL, Johnson DL. Commercial aspects of oocyte retrieval and in vitro fertilization (IVF) for embryo production in problem cows. Theriogenology 1994; 41: 67-72.

19. Massip A, Mermillod P, Dinnyes A. Effects of dilution procedure and culture conditions after thawing on survival of frozen bovine blastocysts produced in vitro. J Reprod Fertil 1993; 97: 65-69.

20. Massip A, Mermillod P, Dynnes A. Morphology and biochemistry of in vitro-produced bovine embryos: implications for their cryopreservation. Human Reprod 1995; 10(11): 3004-3011.

21. Massip A, Mermillod P, Van Langendonckt A, Dynnes A. Veaux issus d'embryons produits in vitro, non congelés ou congelés. Annales de Médécine Vétérinaire 1994; 138 : 333-338

22. McGrath J, Solter D. Nuclear transplantation in the mouse embryo using microsurgery and cell fusion. Science 1983; 220: 1300-1302.

23. Menck C, Guyader-Joly C, Peynot N, LeBourhis D, Lobo RB, Renard JP, Heyman Y. Beneficial effect of Vero cells for developing IVF eggs in two different coculture systems. Reprod Nutr Dev 1997; 37: 141-150.

24. Nagashima H, Cameron DA, Kuwayama M, Young M, Beebe L, Blackshaw W. Nottle MB. Survival of porcine delipated oocytes and embryos after cryopreservation by freezing or vitrification. J Reprod Dev 1999; 45 (2): 167-176

25. Nagashima H, Kashiwazaki N, Ashman RJ, Grupen CG, Nottle MB. Cryopreservation of porcine embryos. Nature 1995; 374: 416.

26. Nagashima H, Kashiwazaki N, Ashman R J, Grupen CG, Seamark RF, Nottle MB Removal of cytoplasmic lipids enhances the tolerance of porcine embryos to chilling. Biol Reprod 1994; 51: 618-622.

27. Nagashima H, Kashiwazaki N, Ashman RJ, Nottle MB. Succesful cryopreservation of porcine early cleavage stage embryos following removal of cytoplasmic lipid droplets. Theriogenology, 1995; 45: 180 abstr.

28. Nagashima H, Kuwayama M, Grupen CG, Ashman RJ, Nottle MB. Vitrification of porcine early cleavage stage embryos and oocytes after removal of cytoplasmic lipid droplets. Theriogenology 1996; $45(1): 180$ abstr.

29. Norberg HS. Ultrastructural aspects of the preattached pig embryo: cleavage and early blastocyst stages. Z Anat Entwicklungsgesch 1973; 143: 95-114.

30. Pangestu $M$, Lewis I, Shaw J. Chilling and cryopreservation of delipated in vitro produced bovine pronuclear-stage embryos. Proc 13th International Congress on Animal Reproduction, Sydney. 1996. p: 15/17.

31. Pollard JW, Leibo SP. Chilling sensitivity of mammalian embryos. Theriogenology 1994;41:101-106.

32. Quinn PJ. A lipid-phase separation model of low temperature damage to biological membranes. Cryobiology 1985; 22: 128-146.

33. Spector AA, Mathur SN, Kaduce TL, Hyman BT. Lipid nutrition and metabolism of cultured mammalian cells. Prog Lipid Res 1981; 19 : 155-186.

34. Spector AA, Yorek MA. Membrane lipid composition and cellular function. J Lipid Res 1985; 26: 1015-1035. 
35. Stubbs $\mathrm{CD}$, Smith $\mathrm{AD}$. The modification of mammalian membrane polyunsaturated fatty acid composition in relation to membrane fluidity and function. Biochimica et Biophysica Acta 1984; 779: 89-137.

36. Thompson JG. Comparison between in vivo derived and in vitro produced pre-elongation embryos from domestic ruminants. Reprod Fertil Dev 1997; 9: 341-354.

37. Thompson JG, Gardner DK, Pug, PA, McMillan WH, Tervit HR. Lamb birth weight following transfer is affected by the culture system used for pre-elongation development embryos. J Reprod Fertil 1994; Abstract Series 13: 25.

38. Ushijima H, Yamakawa H, Nagashima H. Cryopreservation of bovine IVM/IVF embryos at early cleavage stage following removal of cytoplasmic lipid droplets. Theriogenology 1996; 45 (1): 159 abstr.

39. Van Blerkom J, Runner MN. Mitochondrial reorganization during resumption of arrested meiosis in the mouse oocyte. Am J Anat 1984; 171: 335-355.

40. Van Langendonckt A, Auquier P, Donnay I, Massip A, Dessy F. Acceleration of in vitro bovine embryo development in the presence of fetal calf serum. Theriogenology 1996; 45 (1) 194 abstr.

41. Watson PF, Morris GJ. Cold shock injury in animal cells. In: Temperature and Animal Cells (K Bowler and BJ Fuller, Eds.), 1987; 311-340.

42. Wurth YA, Reinders JMC, Rall WF, Kruip AM. Developmental potential of in vitr produced bovine embryos following cryopreservation and single embryo transfer Theriogenology 1994; 42: 1275-1284. 